\title{
Effect of Body Characteristics on Thoracic Attenuation of Intra-Cardiac Electrical Activity Recorded on the Surface Electrocardiogram
}

\author{
J Ng, H Subacius, D Johnson, AH Kadish, JJ Goldberger \\ Northwestern University, Evanston, IL, USA
}

\begin{abstract}
The objective of this study was to determine the effect of body characteristics on the electrical transfer function of the human thorax.

Twenty patients undergoing electrophysiologic study were enrolled. Unipolar stimuli were applied in the right ventricular apical and posterioseptal areas. XYZ surface ECGs and unipolar intra-cardiac electrograms of the pacing impulses were recorded and signal-averaged. The intracardiac-to-surface transfer functions were modeled and compared with body characteristics.

Physical dimensions, respiratory measures obtained by spirometry, and age correlated with magnitude intercepts and slopes of the modeled transfer functions in all three directions. The phase transfer functions were correlated with physical dimensions in the $Y$ and $Z$ directions.

The effect of body characteristics on the frequency dependency of attenuation and phase has implications on the use of surface ECG and body surface mapping.
\end{abstract}

\section{Introduction}

Surface electrocardiography has become an invaluable tool in the diagnosis and treatment of a variety of cardiovascular disorders. Modifications of the standard technique have been used for a number of other applications, such as signal-averaged electrocardiography [1] and body surface mapping [2]. In addition, great effort has been invested in seeking the solution to the "inverse problem", which seeks to define the cardiac epicardial potential distribution based on the body surface potential distribution [3].

An implicit assumption has been made that the surface ECG reflects cardiac electrical activity without distortion. A requirement for this assumption is that the impedance of the human thorax be primarily resistive $[4,5]$ and that capacitive effects of tissue are negligible [6]. The objective of this study is to test this assumption by examining the frequency characteristics of the transfer function of intra-cardiac signals on the surface ECG and to determine whether the frequency response is dependent on body characteristics.

\section{Methods}

\subsection{Patients}

Twenty patients undergoing electrophysiology studies were enrolled in the protocol. There were 14 males and 6 females with a mean age of $55.4 \pm 15.4$ (range 18 to 79 ) years. Physical dimensions measured include height (HT), weight (WT), body mass index (BMI), chest circumference $(\mathrm{CC})$, chest height $(\mathrm{CH})$, chest width $(\mathrm{CW})$. Respiratory measures obtained from spirometry include forced expiratory volume in 1 second (FEV1) and forced vital capacity (FVC). The range and means of these clinical characteristics of the patients are listed in Table 1. All patients provided written, informed consent for this study, which was approved by the Institutional Review Board of Northwestern University.

Table 1. Patient characteristics.

\begin{tabular}{|l|l|l|l|}
\hline Characteristic & Min & Max & Mean \pm SD \\
\hline HT $(\mathrm{cm})$ & 152 & 188 & $173 \pm 10$ \\
\hline WT $(\mathrm{kg})$ & 50 & 127 & $80 \pm 17$ \\
\hline BMI & 19 & 38 & $26 \pm 5$ \\
\hline CC $(\mathrm{cm})$ & 83 & 128 & $106 \pm 13$ \\
\hline CH $(\mathrm{cm})$ & 15 & 32 & $22 \pm 5$ \\
\hline CW $(\mathrm{cm})$ & 27 & 40 & $32 \pm 3$ \\
\hline FEV $1(\mathrm{~mL})$ & 1100 & 4800 & $2647 \pm 883$ \\
\hline FVC $(\mathrm{mL})$ & 2300 & 5200 & $3330 \pm 838$ \\
\hline
\end{tabular}

\subsection{Study protocol}

Upon arrival to the electrophysiology laboratory and after thorough cleansing and mild abrasion of the skin, $\mathrm{Ag} / \mathrm{AgCl}$ adhesive electrodes were applied in a standard Frank XYZ lead configuration. Multiple electrode catheters were positioned in the heart, as clinically indicated, through sheaths placed in the femoral vein. After completion of the diagnostic portion of the test, a quadripolar catheter with $5 \mathrm{~mm}$ interelectrode spacing was positioned in the right ventricular apex (RVA), an anterior and apical site. A second steerable, quadripolar catheter with $5 \mathrm{~mm}$ interelectrode spacing was positioned 
in the right ventricle in the posteroseptal area (PS), a posterior and basal site. Unipolar recordings (band pass filters between $0.05-400 \mathrm{~Hz}$ ) were made from the distal electrode of each catheter with a skin electrode on the thigh as the indifferent electrode. Unipolar stimulation was performed with a programmable stimulator (Bloom Associates, Reading, PA) using a constant voltage stimulus isolation unit from the second pole of each catheter ( $5 \mathrm{~mm}$ from the recording site).

The stimulation protocol was designed so that $0.1 \mathrm{~ms}$ rectangular stimuli with amplitudes of one volt would be applied during a portion of the cardiac cycle with no spontaneous electrical activity. Additionally, the applied signal did not result in ventricular capture in any case because it was not in direct contact with the myocardium. Thus, endocardial recordings from near the site of stimulation ( $5 \mathrm{~mm}$ away) were made simultaneously with the surface electrocardiographic recordings in order to be able to measure the body's attenuation of this signal. These measurements were made from the RVA and PS.

During each five minute pacing period, the $\mathrm{X}, \mathrm{Y}$ and $\mathrm{Z}$ electrocardiographic data and the unipolar recordings from the distal pole of the catheter from which the stimuli were applied (5 mm away) were recorded on a commercially available system (Predictor I, Arrhythmia Research Technology, Austin, Texas) at a sampling frequency of $2000 \mathrm{~Hz}$. In addition, a square wave synchronous to the pacing stimulus was recorded from the Bloom stimulator.

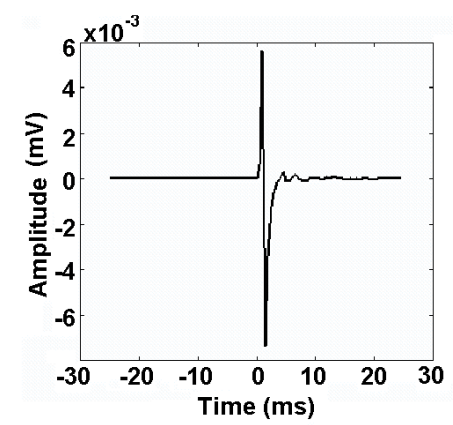

Figure 1. Signal-averaged impulse following baseline correction, zeroing of the first $25 \mathrm{~ms}$, and application of a $50 \mathrm{~ms}$ Hanning window.

\subsection{Signal processing}

Digital signal processing was performed using analysis software developed with MATLAB (The Mathworks Inc., Natick, MA). Using the square wave as the fiducial point, time based signal averaging of the applied stimulus was performed to reduce noise levels. A $50 \mathrm{~ms}$ segment centered around the fiducial point was selected for analysis. Baseline correction was performed using a third- order polynomial trend removal algorithm. With the assumption of a causal system, the first $50 \mathrm{~ms}$ of the signal was replaced with zeroes. A Hanning window was then applied and the data was zero-padded to 400 points. An illustration of the result of these maneuvers is shown in figure 1.

The frequency domain representation of these signals was calculated using the Fast Fourier Transform with a frequency resolution of $5 \mathrm{~Hz}$. The frequency range of 25$250 \mathrm{~Hz}$ was chosen for analysis to avoid baseline wander. The $\mathrm{X}, \mathrm{Y}$, and $\mathrm{Z}$ magnitude transfer functions were calculated by dividing the magnitude of the Fourier transform of the electrocardiographic recording by the magnitude of the Fourier transform of the intracardiac recording:

$$
\begin{aligned}
& \left|H_{X}(f)\right|=|X(f)| /|I C(f)| \\
& \left|H_{Y}(f)\right|=|Y(f)| /|I C(f)| \\
& \left|H_{Z}(f)\right|=|Z(f)| /|I C(f)|
\end{aligned}
$$

The phase transfer functions were calculated by subtracting the unwrapped phase angle of the Fourier transform of the intracardiac recording from the unwrapped phase angle of the electrocardiographic recording:

$$
\begin{aligned}
& \operatorname{ang}\left(H_{X}(f)\right)=\operatorname{ang}(X(f))-\operatorname{ang}(I C(f)) \\
& \operatorname{ang}\left(H_{Y}(f)\right)=\operatorname{ang}(Y(f))-\operatorname{ang}(I C(f)) \\
& \operatorname{ang}\left(H_{Z}(f)\right)=\operatorname{ang}(Z(f))-\operatorname{ang}(I C(f))
\end{aligned}
$$

\subsection{Data analysis and statistics}

For each subject, two sets of three magnitude and three phase transfer functions were generated from the right ventricular stimulation/recordings and the posteroseptal stimulation/recordings. If the signal averaging and other data processing did not adequately reduce noise levels or introduced obvious artifacts, the data for that particular lead was excluded from analysis. The magnitude transfer function were expressed in decibels and plotted on a logarithmic frequency scale. The phase transfer functions were expressed in radians and plotted in a linear scale.

The data were analyzed with a multilevel mixed effect regression models using HLM 6.0 software by Scientific Software International. Maximum likelihood estimation was used to obtain slope-and-intercept models for the individual magnitude and phase functions. These individual slopes and intercepts, in turn, were used to investigate the explanatory power of patient-level characteristics listed above. Patient variables were entered into the regression model in a step-wise fashion. Strongest predictors were entered first followed by additional predictors who had significant partial correlations. If the significance for a variable already in the model dropped below .10, it was removed from a final 
model. Therefore, patient-level variables in the final model represent an optimal subset.

First, frequency dependent attenuation and phase shift for each of the transfer functions was evaluated. We compared the differences: 1) among X, Y, and Z transfer functions averaged across RVA and PS sites and 2) transfer functions obtained for each lead from the right ventricular apex against those obtained from the posteroseptal area. Second, parameters that were computed to characterize magnitude and phase transfer functions were used as outcomes to be predicted by patients' body characteristics. Differences that exceeded $\mathrm{P}<0.05$ were considered statistically significant.

\section{Results}

\subsection{Transfer function modeling}

The X, Y, and Z transfer functions could be calculated for 14, 18, and 14 patients, respectively, for recordings made from the RVA and 14, 18, and 16 patients, respectively, for recordings made from the PS. The mean transfer functions, which are shown figure 2, showed greater attenuation at lower frequencies and a primarily linear phase response.
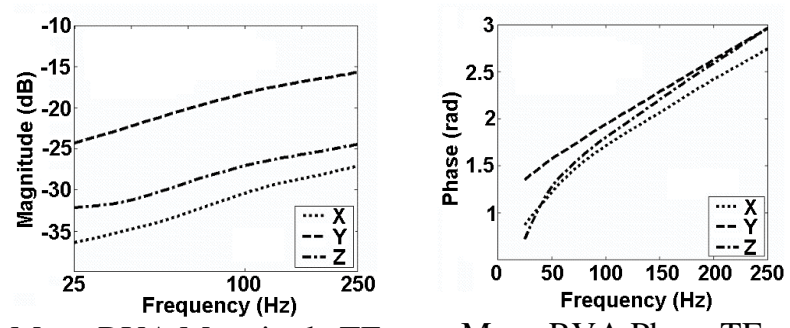

Mean RVA Magnitude TF

Figure 2. The mean XYZ magnitude RVA transfer functions (left) and the mean XYZ RVA phase transfer functions (right).

Table 2 shows the mean, standard deviations, and the $95 \%$ plausible value intervals of the modeled transfer function characteristics. The modeled magnitude transfer function for each lead was summarized with two variables: the intercept at $250 \mathrm{~Hz}$ expressed in decibels and the slope expressed as decibels per decade of frequency in $\mathrm{Hz}$. The modeled phase transfer function was summarized by the slope in radians per $\mathrm{Hz}$.

\subsection{Comparison of intraindividual $\mathrm{X}, \mathrm{Y}$, and $\mathrm{Z}$ transfer functions}

The intraindividual modeled $\mathrm{X}, \mathrm{Y}$, and $\mathrm{Z}$ transfer functions were compared by lead and impulse location. The magnitude slopes of the transfer functions recorded in Lead Z were larger than those of Lead Y $(\mathrm{P}=0.04)$ in

the RVA recordings. There were no significant differences in magnitude slopes between leads the PS recordings. There were no differences in magnitude slopes between the RVA and PS recordings.

The magnitude intercepts at $250 \mathrm{~Hz}$ showed significant differences between all leads $(\mathrm{P}<0.003)$, except between leads $\mathrm{X}$ and $\mathrm{Z}$ from the PS, which showed only a trend ( $\mathrm{P}$ $=0.06)$. Lead $\mathrm{Y}$ showed an increase in magnitude intercept from the RVA to the PS $(P=0.006)$, while lead $\mathrm{Z}$ showed a decrease $(\mathrm{P}<0.001)$.

The intraindividual transfer function phase slopes were also compared. In the RVA, the phase slope of Lead $\mathrm{Z}$ was significantly larger than those of Lead Y $(p<0.001)$ and those of Lead $\mathrm{X}(\mathrm{p}<0.001)$ with no difference between Leads $X$ and $Y$. In the PS, only a difference between Leads $\mathrm{Z}$ and $\mathrm{Y}(\mathrm{p}<0.001)$ was found. There was no significant change in phase slope moving the impulse location from the RVA to the PS.

Table 2. Transfer function characteristics.

\begin{tabular}{lllll} 
& & & \multicolumn{2}{c}{$95 \%$ CI } \\
Magnitude & Mean & SD & Lower & Upper \\
X int (dB) & -27.649 & 7.230 & -41.819 & -13.478 \\
X slope & 5.385 & 5.186 & -4.780 & 15.549 \\
(dB/decade) & & & & \\
Y int (dB) & -14.289 & 3.725 & -21.590 & -6.989 \\
Y slope & 3.703 & 5.202 & -6.492 & 13.898 \\
(dB/decade) & & & & \\
$\begin{array}{l}\text { Z int (dB) } \\
\text { Z slope } \\
\text { (dB/decade) }\end{array}$ & -30.735 & 5.890 & -42.279 & -19.191 \\
$\begin{array}{l}\text { Phase } \\
\text { X slope } \\
\text { (rad/Hz) }\end{array}$ & 0.941 & 6.625 & -6.043 & 19.926 \\
$\begin{array}{l}\text { Y slope } \\
\text { (rad/Hz) }\end{array}$ & 0.010 & 0.002 & 0.005 & 0.014 \\
$\begin{array}{l}\text { Z slope } \\
\text { (rad/Hz) }\end{array}$ & 0.015 & 0.003 & 0.010 & 0.020 \\
\end{tabular}

\subsection{Effect of clinical characteristics on transfer functions}

Group differences in the magnitude transfer characteristics were optimally explained by the patients' physical dimensions, respiratory measures, and age. Once patients' physical dimensions were entered in the regression model for differences in the phase transfer functions, respiratory characteristics and age could not predict residual variance. Table 3 lists the patient characteristics which had effect on the magnitude and phase transfer functions.

Table 3. Influence of patient characteristics on the 
magnitude transfer function.

$\begin{array}{llll}\text { Mag } & \text { Pt Char } & \text { Coefficient } & \text { P-value } \\ \text { X int } & \text { FVC } & -3.5 \times 10^{-3} \mathrm{~dB} / \mathrm{mL} & 0.013 \\ \text { X slope } & \text { Age } & -0.22 \mathrm{~dB} / \mathrm{dec} / \mathrm{yr} & <0.001 \\ \text { Y int } & \text { Age } & -0.15 \mathrm{~dB} / \mathrm{yr} & 0.001 \\ & \text { CC } & -0.13 \mathrm{~dB} / \mathrm{cm} & 0.004 \\ & \text { FEV1 } & -2.9 \times 10^{-3} \mathrm{~dB} / \mathrm{mL} & 0.001 \\ \text { Y slope } & \text { HT } & -0.31 \mathrm{~dB} / \mathrm{dec} / \mathrm{cm} & 0.004 \\ & \text { FEV1 } & 4.5 \times 10^{-3} \mathrm{~dB} / \mathrm{dec} / \mathrm{mL} & 0.002 \\ \text { Z int } & \text { CH } & -0.8 \mathrm{~dB} / \mathrm{cm} & <0.001 \\ \text { Z slope } & \text { FEV1 } & 2.6 \times 10^{-3} \mathrm{~dB} / \mathrm{dec} / \mathrm{mL} & <0.001 \\ \text { Phase } & & & \\ \text { Y slope } & \text { HT } & 1.3 \times 10^{-4} \mathrm{rad} / \mathrm{Hz} / \mathrm{cm} & 0.007 \\ \text { Z slope } & \text { CC } & -1.2 \times 10^{-4} \mathrm{rad} / \mathrm{Hz} / \mathrm{cm} & 0.014\end{array}$

\section{Discussion and conclusions}

In this study, the frequency dependency on electric attenuation of intra-cardiac signals was demonstrated from surface ECG recordings of pacing impulses applied in the right ventricular apical and the posteroseptal area of the right ventricle. The linear modeling of the magnitude transfer functions showed that lower frequencies were attenuated more than higher frequencies. These findings suggest that the thorax has both capacitive as well as resistive effects on electrical attenuation.

Analysis of the patients' clinical characteristics showed that physical dimensions, respiratory measures and age had effects on the transfer function characteristics. Other studies using signal-averaged ECG have shown the dependency of QRS duration on body mass index [7-8], gender [8-9], and posture [10].

The results of this study have several implications on traditional ECG analysis. The key features in the ECG, which include the P wave, QRS complex, S-T segment, and $T$ wave, each have specific frequency ranges that contain the most information of the respective signals. The utility of analyzing the timing, amplitude, and morphology of these features has been well demonstrated. The determined linearity of the phase transfer function suggests that timing measures such as P-R interval or Q-T interval should not be affected by differences in thoracic impedance, despite the differences in frequency content of the $\mathrm{P}$ wave, QRS complex, and T wave. For amplitude measurements, however, the frequency dependency of thoracic attenuation will have an effect, especially if amplitude of one ECG feature is measured relative to that of another.

The implication on body surface mapping and the inverse solution is also evident. The assumption of a resistive network allows the modeling of the surface response as a linear combination of local activations without having to consider frequency dependant attenuation or propagation delays. However, this study showed that body characteristics affect both of these things and should be considered when modeling intracardiac electrical activity on the surface.

\section{References}

[1] Dunbar DN, Denes P. Operational aspects of signalaveraged electrocardiography. Progress in Cardiovascular Diseases 1993;5:329-348.

[2] Oster HS, Taccardi B, Lux RL, Ershler PR, Rudy Y. Noninvasive electrocardiographic imaging: reconstruction of epicardial potentials, electrograms, and isochrones and localization of single and multiple electrocardiac events. Circulation 1997;96:1012-1024.

[3] Rudy Y, Messinger-Rapport BJ. The inverse problem in electrocardiography: solutions in terms of epicardial potentials. Crit Rev Biomed Eng 1988;16:215-268.

[4] Rush S, Abildskov JA, McFee R. Resistivity of body tissues at low frequencies. Circ Res 1963;12:40-50.

[5] Schwan HP, Kay CF. The conductivity of living tissues. Ann N Y Acad Sci 1956;65:1007-1013.

[6] Schwan HP, Kay CF. Capacitive properties of body tissues. Circ Res 1957;5:439-443.

[7] Iwasaka T, Usui H, Masui A, Tamura K, Sugiura T, Karakawa M, Inada M. Effect of body characteristics on the variables of signal-averaged electrocardiogram in healthy teenage subjects. Cardiology 1993;83:71-5.

[8] Masui A, Tsuji H, Tamura K, Tarumi N, Sugiura T, Iwasaka T, Inada M. Effect of body characteristics on the variables of signal-averaged electrocardiograms in healthy subjects. Chest 1994;105:1357-59.

[9] Timmermans C, Ector H, Haisty KW, Hammill SC, Kienzle MG, Ozawa Y, Reddy BR, Underwood DA. Signal-averaged ECG parameters in cardiac normals using Frank lead system and Fourier transform filter and gender specific differences: a multicenter study. Pacing Clin Electrophysiol. 1994;17:303-11.

[10] Kautzner J, Hartikainen JEK, Camm AJ, Marek M. Effect of upright posture on filtered QRS parameter of the signalaveraged electrocardiogram in healthy volunteers. Am Heart J 1997; 134:1002-4.

Address for correspondence

Jeffrey J. Goldberger, MD

Northwestern University Feinberg School of Medicine

Division of Cardiac Electrophysiology

251 East Huron, Feinberg Pavilion 8-542

Chicago, IL 60611, USA

Email: j-goldberger@northwestern.edu 